(C) by R. Oldenbourg Verlag, München

\title{
Crystal structure of the clathrates $R_{8} \mathrm{In}_{8} \mathrm{Ge}_{38}$ and $\mathrm{K}_{8} \mathrm{In}_{8} \mathrm{Ge}_{38}$
}

\author{
H. G. von Schnering, H. Menke, R. Kröner, E.-M. Peters, K. Peters
}

Max-Planck-Institut für Festkörperforschung. Heisenbergstraße 1, D-70569 Stuttgart, Germany

and R. Nesper

ETH Zürich. Laboratorium für Anorganische Chemie, Universitätsstr. 6, CH-8092 Zürich, Switzerland

Received March 3, 1998, transferred to Ist update of database ICSD in 1999, CSD-No. 409253 and CSD-No. 409254
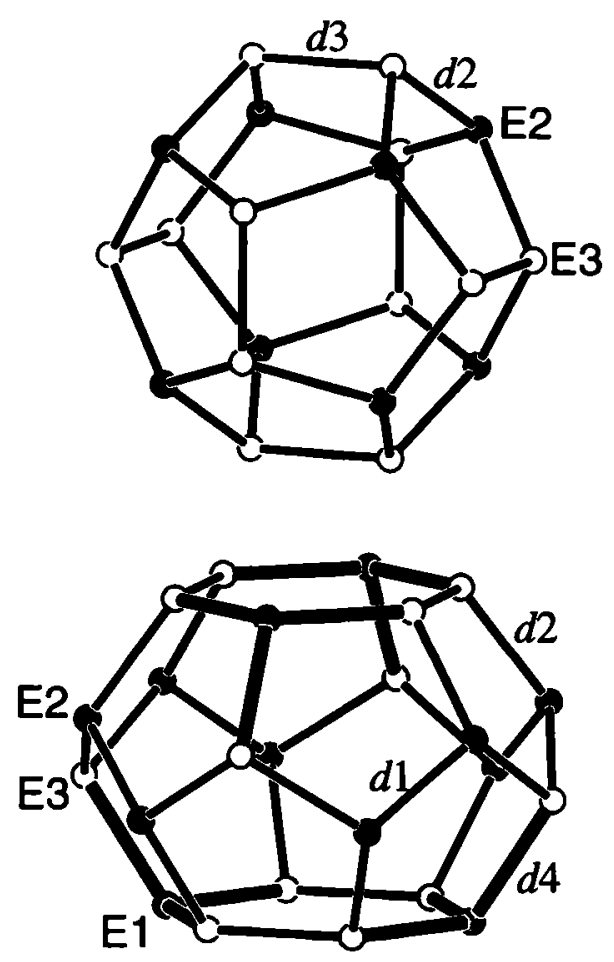

\section{RbsIngGe38}

Source of material: Stoichiometric mixtures of the elements were sealed in $\mathrm{Nb} / \mathrm{Ta}$ capsules under $\mathrm{Ar}$, heated up to $1270 \mathrm{~K}(3 \mathrm{~h})$, annealed at $970 \mathrm{~K}$ (2-3 d) and colled down. RbsIngGe38 forms small crystals. The grey brittle compound is a semiconductor, stable in air and against dilute acids and bases (see ref. 1).

The refinement of the site occupancies results in the composition RbsIn $_{n} G_{e} 46-n$ with $n=7.82 \pm 0.46$. Bond lengths in the $E_{46}$ net (figure): $d 1=2.525(1) \AA, d 2=2.519(1) \AA, d 3=2.568(1) \AA, d 4$ $=2.641(1) \AA$.

Ge38In\&Rb\&, cubic, $P m \overline{3} n$ (No. 223), $a=11.033$ (2) $\AA, V=1343.0 \AA^{3}$, $Z=1, R(F)=0.048, R_{\mathrm{w}}(F)=0.031$.

Table 1. Parameters used for the X-ray data collection

\begin{tabular}{ll}
\hline Crystal: & grey, metallic luster, size $0.1 \times 0.1 \times 0.15 \mathrm{~mm}$ \\
Wavelength: & Mo $K_{\alpha}$ radiation $(0.71069 \AA)$ \\
$\mu::$ & $305.6 \mathrm{~cm}^{-1}$ \\
Diffractometer: & Nicolet R3m/V \\
Scan mode: & $\omega$ \\
$T_{\text {measurement: }}$ & $293 \mathrm{~K}$ \\
$20_{\text {max }}:$ & $55^{\circ}$ \\
$N(h k l)_{\text {unique: }}:$ & 241 \\
Criterion for $I_{0}:$ & $I_{0}>3 \sigma\left(I_{0}\right)$ \\
N(param $)_{\text {refined: }}$ & 19 \\
Programs: & SHELXS-86, SHELX-76
\end{tabular}

Table 2. Final atomic coordinates and displacement parameters (in $\AA^{2}$ )

\begin{tabular}{|c|c|c|c|c|c|c|c|c|c|c|c|}
\hline Atom & Site & Occ. & $x$ & $y$ & $z$ & $U_{11}$ & $U_{22}$ & $U_{33}$ & $U_{12}$ & $U_{13}$ & $U_{23}$ \\
\hline $\mathrm{Rb}$ l & $2 a$ & & 0 & $\mathbf{0}$ & 0 & $0.015(1)$ & $U_{11}$ & $U_{11}$ & 0 & 0 & 0 \\
\hline $\mathrm{Rb} 2$ & $6 d$ & & $1 / 4$ & $1 / 2$ & 0 & $0.023(2)$ & $0.035(2)$ & $U_{22}$ & 0 & 0 & 0 \\
\hline $\mathrm{E} 1(\mathrm{In})$ & $6 c$ & $0.89(1)$ & $1 / 4$ & 0 & $1 / 2$ & $0.015(1)$ & $0.0103(9)$ & $U_{22}$ & 0 & 0 & 0 \\
\hline $\mathrm{E} 1(\mathrm{Ge})$ & $6 c$ & 0.11 & $1 / 4$ & 0 & $1 / 2$ & 0.015 & 0.0103 & $U_{22}$ & 0 & 0 & 0 \\
\hline E2(In) & $16 i$ & 0.02 & 0.1839 & $x$ & $x$ & 0.0116 & $U_{11}$ & $U_{11}$ & $-0.0010(4)$ & $U_{12}$ & $U_{12}$ \\
\hline $\mathrm{E} 2(\mathrm{Ge})$ & $16 i$ & $0.98(1)$ & $0.1839(1)$ & $x$ & $x$ & $0.0116(6)$ & $U_{11}$ & $U_{11}$ & $-0.0010(4)$ & $U_{12}$ & $U_{12}$ \\
\hline E3(In) & $24 k$ & 0.09 & 0 & 0.3013 & 0.1164 & 0.0114 & 0.0117 & 0.0110 & 0 & 0 & -0.0008 \\
\hline E3(Ge) & $24 k$ & $0.91(1)$ & 0 & $0.3013(1)$ & $0.1164(1)$ & $0.0114(8)$ & $0.0117(8)$ & $0.0110(8)$ & 0 & 0 & $-0.0008(7)$ \\
\hline
\end{tabular}




\section{K8IngGe38}

Source of material: For the synthesis and the chemical properties see above (Rb8In8Ge38). K8In8Ge38 is also a semiconductor. The grey brittle crystals are small (see ref. 1). Reactions with different In:Ge ratios gave no indications for a homogeneity range (powder pattern) in contrast to ref. 2.

The refinement of the site occupancies results in the composition $\mathrm{K}_{8} \mathrm{In}_{n} \mathrm{Ge}_{46-n}$ with $n=8.14 \pm 0.62$. Bond lengths in the $\mathrm{E}_{46}$ net (figure): $d 1=2.528$ (1) $\AA, d 2=2.511$ (1) $\AA, d 3=2.536(1) \AA, d 4$ $=2.642(1) \AA$.

Ge38InsK8, cubic, $P m \overline{3} n$ (No. 223), $a=10.997(2) \AA, V=1329.9 \AA^{3}$ $Z=1, R(F)=0.035, R_{w}(F)=0.027$.

Table 3. Parameters used for the $\mathrm{X}$-ray data collection

Crystal:

Wavelength:

$\mu:$

Diffractometer:

Scan mode:

Teasurement:

$2 \theta_{\max }$

$\mathrm{N}(h k l)_{\text {unique: }}$

Criterion for 10

$\mathrm{N}(\text { param })_{\text {refined: }}$

Programs: grey, metallic luster, size $0.1 \times 0.15 \times 0.2 \mathrm{~mm}$

Mo $K \alpha$ radiation $(0.71069 \AA)$

$243.2 \mathrm{~cm}^{-1}$

Nicolet R3m/V

$\omega$

$293 \mathrm{~K}$

$55^{\circ}$

237

$l_{0}>3 \sigma\left(I_{0}\right)$

19

SHELXS-86. SHELXS-76

Table 4. Final atomic coordinates and displacement parameters (in $\AA^{2}$ )

\begin{tabular}{llllllllllll}
\hline Atom & Site & Occ. & $x$ & $y$ & $z$ & $U_{11}$ & $U_{22}$ & $U_{33}$ & $U_{12}$ & $U_{13}$ & $U_{23}$ \\
\hline K1 & $2 a$ & & 0 & 0 & 0 & $0.021(2)$ & $U_{11}$ & $U_{11}$ & 0 & 0 & 0 \\
K2 & $6 d$ & & $1 / 4$ & $1 / 2$ & 0 & $0.031(3)$ & $0.049(3)$ & $U_{22}$ & 0 & 0 & 0 \\
EI(In) & $6 c$ & $0.93(1)$ & $1 / 4$ & 0 & $1 / 2$ & $0.0155(8)$ & $0.0129(6)$ & $U_{22}$ & 0 & 0 & 0 \\
E1(Ge) & $6 c$ & 0.07 & $1 / 4$ & 0 & $1 / 2$ & 0.0155 & 0.0129 & $U_{22}$ & 0 & 0 & 0 \\
E2(In) & $16 i$ & 0.04 & 0.1836 & $x$ & $x$ & 0.0134 & $U_{11}$ & $U_{11}$ & -0.0006 & $U_{12}$ & $U_{12}$ \\
E2(Ge) & $16 i$ & $0.96(2)$ & $0.1836(1)$ & $x$ & $x$ & $0.0134(4)$ & $U_{11}$ & $U_{11}$ & $-0.0006(3)$ & $U_{12}$ & $U_{12}$ \\
E3(In) & $24 k$ & 0.08 & 0 & 0.3010 & 0.1153 & 0.0137 & 0.0135 & 0.0135 & 0 & 0 & -0.0005 \\
E3(Ge) & $24 k$ & $0.92(1)$ & 0 & $0.3010(1)$ & $0.1153(1)$ & $0.0137(6)$ & $0.0135(6)$ & $0.0135(6)$ & 0 & 0 & $-0.0005(4)$
\end{tabular}

\section{References}

1. Kröner, R.: Zintl-Phasen der Alkalimetalle und des Bariums mit Clathratstruktur. Dissertation, Universität Stuttgart, 1989.

2. Westerhaus, W.; Schuster, H.-U.: Ternäre Phasen mit modifizierter K8Ge46-Käfigstruktur. Z. Naturforsch. 32b (1977) 1365-1367.
3. Sheldrick, G. M.: Phase Annealing in SHELX-90: Direct Methods for Large Structures. Acta Crystallogr. A 46 (1990) 467-473.

4. Sheldrick, G. M.: SHELX-76, Programs for Crystal Structures Determination. Cambridge 1976 